\title{
CARACTERÍSTICAS DOS DETERMINANTES SOCIAIS EM SAÚDE: CAMINHONEIROS EM UMA RODOVIA DO BRASIL
}

\section{ARTIGO ORIGINAL}

REGAZZI, Isabel Cristina Ribeiro ${ }^{1}$

REGAZZI, Isabel Cristina Ribeiro. Características dos determinantes sociais em saúde: Caminhoneiros em uma rodovia do Brasil. Revista Científica Multidisciplinar Núcleo do Conhecimento. Ano 05, Ed. 11, Vol. 07, pp. 45-56. Novembro de 2020. ISSN: 2448-0959, Link de acesso: https://www.nucleodoconhecimento.com.br/saude/caracteristicas-dos-determinantes

\section{RESUMO}

O presente estudo direcionou-se a atender a seguinte questão norteadora: Quais as características de alguns dos determinantes sociais a saúde dos caminhoneiros de uma rodovia Federal do Brasil? Desta forma, foi realizado um levantamento das características de alguns dos determinantes sociais a saúde dos caminhoneiros de uma rodovia do estado Rio de Janeiro (RJ), Brasil. Trata-se de um estudo quantitativo, exploratório e de corte transversal. Os dados foram coletados por meio de um formulário contendo perguntas fechadas sobre a saúde física e mental dos caminhoneiros e analisados por técnicas de estatística descritiva. Foi montado um stand junto ao posto da Polícia Federal na rodovia BR 101 no trecho de Casimiro de Abreu - RJ, onde os caminhoneiros eram abordados para realizarem avaliação de saúde e preencherem a pesquisa. Participaram da pesquisa 28 caminhoneiros, nos quais o total era do sexo masculino, sendo que $89,3 \%$ relataram não possuir diabetes mellitus e $86,5 \%$ não apresentaram hipertensão arterial sistêmica. Foi observado que $78,6 \%$ dos caminhoneiros possuem residência fixa no estado do Rio de Janeiro,

\footnotetext{
1 Doutora em ciências da saúde, Professor Associado Universidade Federal Fluminense (UFF).
} 
$71,4 \%$ relataram não possuir plano de saúde, $50 \%$ portavam categoria de habilitação "D" e 35,7\% possuem carga horária de trabalho de 8 horas por dia. Em relação ao tipo de produto transportado $92,8 \%$ desses profissionais relataram não transportar materiais perigosos. Destaca-se que a profissão de caminhoneiro é uma carreira que aumenta a vulnerabilidade dos trabalhadores a doenças e agravos a saúde, visto que as características específicas deste exercício laboral e seu ambiente de trabalho propiciam ao trabalhador a adotar maus hábitos alimentares e de descanso, além de favorecer o sedentarismo, o estresse e a redução da procura por um serviço de atenção primária a saúde.

Palavras-chaves: Saúde do trabalhador, condução de veículo, vulnerabilidade em saúde.

\section{INTRODUÇÃO}

Os caminhoneiros são profissionais tanto assalariados quanto autônomos que prestam serviços às corporações pertencentes aos ramos da logística e das transportadoras. Entre suas diversas funções, os caminhoneiros guincham, removem e entregam mercadorias. Nesta profissão, de acordo com um estudo realizado pela Confederação Nacional de Transporte, nas capitais das regiões metropolitanas brasileiras, prevalecem trabalhando no transporte de carga os indivíduos do sexo masculino, sendo estes $99,8 \%$ do total (CNT, 2016).

Esses profissionais trabalham conduzindo caminhões e, na maioria das vezes, realizam rotas longas para entregar os pedidos aos clientes com o intuito de obedecer ao período estipulado pela empresa. Dessa maneira, a extensa jornada de trabalho, somada ao uso de drogas "estimulantes", como as substâncias psicoativas visando alcançar o prazo de entrega, pode gerar agravos à saúde de tal classe trabalhadora (ALESSI; ALVES, 2015).

Diversos estudos apontam que, em razão da exaustiva rotina de trabalho, muitos caminhoneiros desenvolvem problemas de saúde associados a hábitos de vida não saudáveis, a citar: o sedentarismo, distúrbios do sono, alterações gastrointestinais 
como o refluxo gastroesofágico, problemas ergonômicos e, em especial, o uso de álcool, constituindo o principal elemento causador de acidente de trânsito entre os caminhoneiros (MASSON; MONTEIRO, 2010). Visando atestar a vulnerabilidade a agravos à saúde decorrente deste tipo de carreira profissional, dentre as fatalidades ocupacionais por acidentes automobilísticos nos Estados Unidos, 46\% dos óbitos foram representados pelos caminhoneiros (CHEN, 2015).

O presente estudo direcionou-se a atender a seguinte questão norteadora: Quais as características de alguns dos determinantes sociais a saúde dos caminhoneiros de uma rodovia Federal do Brasil?

Justifica-se a necessidade desse estudo em razão dos dados encontrados na literatura científica que evidenciam risco a saúde desencadeados pela profissão de caminhoneiro. O exercício dessa profissão exige poucas horas de sono e longos períodos longe de casa, sendo somado ao stress e ansiedade ocasionados pelo trânsito e maus hábitos alimentares. Logo, tais fatos aumentam a vulnerabilidade destes trabalhadores a diversos agravos à saúde, sendo destacadas as Infecções Sexualmente Transmissíveis (IST's), a predisposição a doenças crônicas, os acidentes de trânsito e as doenças emocionais, como a depressão (HINO et al 2017).

Desta forma, o objetivo consistiu em verificar as características de alguns dos determinantes sociais a saúde dos caminhoneiros de uma rodovia do estado Rio de Janeiro (RJ), Brasil.

\section{METODOLOGIA}

O presente estudo tem abordagem quantitativa, exploratória e foi do tipo corte transversal. Avaliou caminhoneiros que transitaram pela Rodovia BR-101, no município de Casimiro de Abreu, localizado no Estado do Rio de Janeiro, Brasil. A coleta de dados ocorreu durante uma ação de promoção à saúde a caminhoneiros, realizada no dia 14 de julho de 2018. A referida ação foi promovida pela Universidade Federal Fluminense (UFF) do Campus de Rio das Ostras, em parceria com a prefeitura municipal de Casimiro de Abreu, e contou com o apoio da Polícia Rodoviária 
Federal (PRF). Um stand foi montado ao lado do posto da polícia federal, em que foram oferecidos serviços de avaliação de pressão arterial, corte de cabelo, e orientações sobre direção defensiva.

A coleta de dados foi baseada em entrevistas realizadas por meio de um formulário, contendo perguntas fechadas sobre os dados socioeconômicos, demográficos e a saúde física e mental dos caminhoneiros. O instrumento foi elaborado previamente a partir de questões existentes no formulário disponibilizado pelo o Instituto Brasileiro de Geografia e Estatística (IBGE) e questões específicas para a referida população, na qual foi pré testada. Sob a orientação dos professores organizadores da ação os alunos de graduação enfermagem aplicaram os questionários no dia da ação. Para cada caminhoneiro participante, aplicou-se o termo de consentimento livre e esclarecido (TCLE). Os critérios de inclusão foram os caminhoneiros (autônomos e de frota) que transitaram pela BR 101 no trecho em Casimiro de Abreu. A amostra da pesquisa foi caracterizada como de conveniência, tendo sido recrutados os caminhoneiros que reduziam a velocidade do veículo a avistar a Polícia Federal, parando no acostamento da rodovia e que no momento demonstravam interesse por participar da ação. A amostragem por conveniência é adequada quando empregada principalmente em pesquisas exploratória, visando a obtenção de resposta de maneira rápida e barata e para geração de ideias (OLIVEIRA, 2001).

Os dados coletados foram digitados em planilha eletrônica e analisados por meio de medidas de tendência central e de proporção. Os dados foram processados no Programa $\mathrm{R}$, que é gratuito e disponível para download. Os resultados encontrados foram representados em gráficos e tabelas.

O presente estudo faz parte do projeto submetido e aprovado ao Comitê de Ética e Pesquisa (CEP) do Hospital Universitário Antônio Pedro (HUAP). Estando à pesquisadora comprometida em seguir todos os princípios e normas préestabelecidos pela Resolução no 466/ 2012 do Conselho Nacional de Saúde (CNS), que regulamenta a pesquisa envolvendo seres humanos (BRASIL, 2012). Aprovado 
pelo Comitê de Ética em Pesquisa (CEP HUAP/UFF), CAAE: 73671617.5.0000.5243 Número do Parecer: 2.384.79.

\section{RESULTADOS}

A amostra da pesquisa foi de 28 caminhoneiros. As principais características sociodemográficas dos participantes foram ser do sexo masculino (100\%) e ter idade mediana de 46 anos, sendo a menor idade observada de 23 anos e a maior de 64 anos (Figura 1). A renda mensal média encontrada foi de $R \$ 2.000,00$, sendo o valor mínimo da renda observado de $\mathrm{R} \$ 1.000,00$ e o máximo de $\mathrm{R} \$ 10.000,00$. O local de residência com maior predomínio foi o estado do Rio de Janeiro, onde $78,6 \%$ dos caminhoneiros possuem moradia fixa (Tabela 1). Verificou-se que o regime de trabalho com maior prevalência foi a carga horária diária de 8 horas, representando $35,7 \%$ dos caminhoneiros participantes e, que $50 \%$ dos caminhoneiros tem a habilitação de categoria "D" (Tabela 2 e Figura 2).

Figura 1 - Boxplot da distribuição da idade dos caminhoneiros na BR 101 - Casimiro de Abreu, 2018. 


\section{Boxplot de Idade}

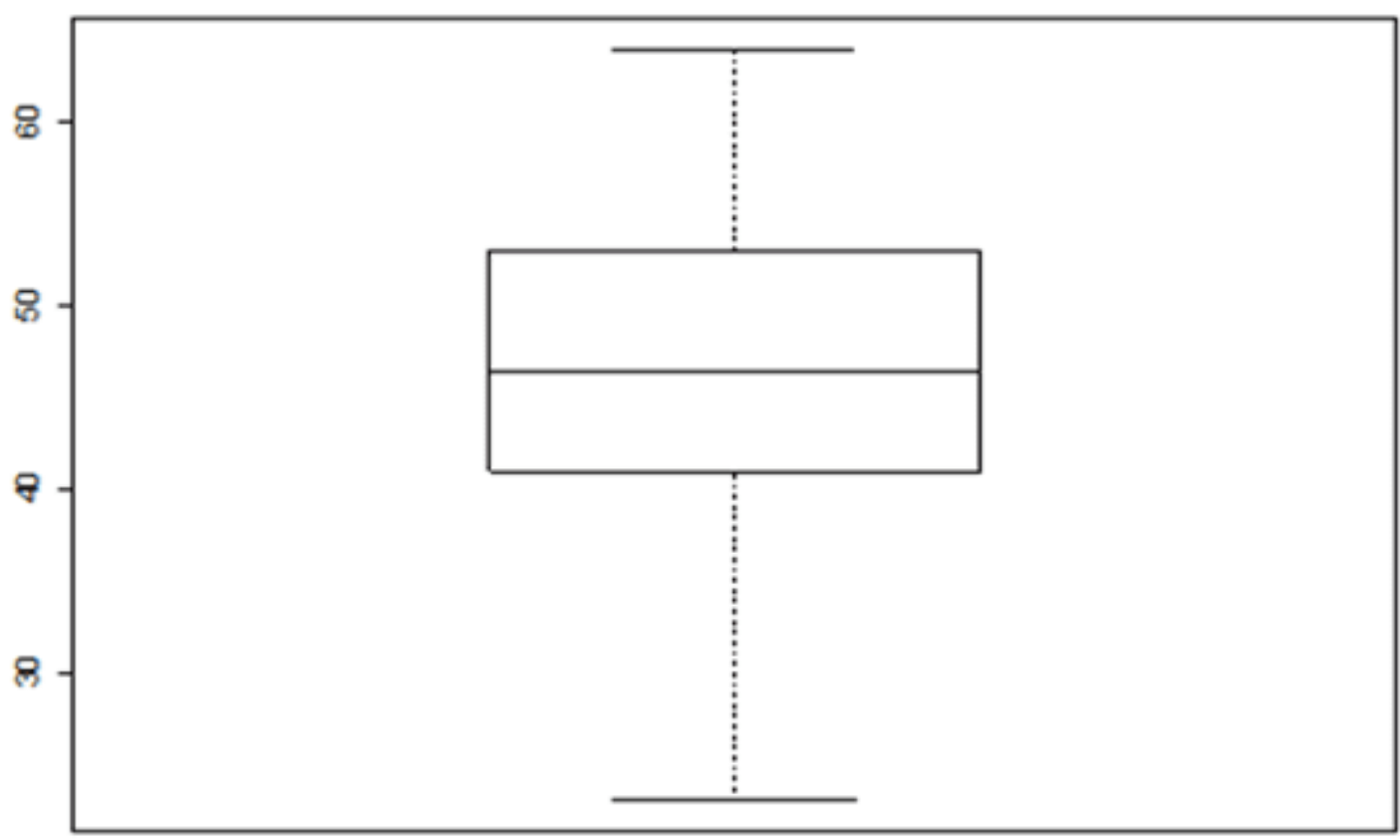

Fonte: arquivo pessoal

Tabela 1 - Distribuição das variáveis relacionadas ao local de residência, dos caminhoneiros, 2018.

\begin{tabular}{|l|l|}
\hline Local de residência & Percentual \\
\hline Espírito Santo & $(\mathrm{N}=28)$ \\
\hline Minas Gerais & $3,6 \%$ \\
\hline Pernambuco & $3,6 \%$ \\
\hline Rio de Janeiro & $3,6 \%$ \\
\hline São Paulo & $78,6 \%$ \\
\hline
\end{tabular}

Fonte: arquivo pessoal

Tabela 2 - Distribuição das variáveis relacionadas carga horária e a categoria de habilitação dos caminhoneiros, 2018. 


\begin{tabular}{|c|c|}
\hline $\begin{array}{ll}\text { Categorias } & \text { de } \\
\text { habilitação } & \end{array}$ & $\begin{array}{l}\text { Percentual } \\
(\mathrm{N}=28)\end{array}$ \\
\hline B & $7,1 \%$ \\
\hline $\begin{array}{l}\text { C } \\
\text { D }\end{array}$ & $\begin{array}{l}17,9 \% \\
50,0 \%\end{array}$ \\
\hline $\mathbf{E}$ & $25,0 \%$ \\
\hline $\begin{array}{l}\text { Carga } \\
\text { horária }\end{array}$ & $\begin{array}{l}\text { Percentual } \\
(\mathrm{N}=28)\end{array}$ \\
\hline 5 a 6 horas & $14,3 \%$ \\
\hline 7 horas & $14,3 \%$ \\
\hline 8 horas & $35,7 \%$ \\
\hline 8,1 a 10 horas & $17,9 \%$ \\
\hline 11 a 12 horas & $14,3 \%$ \\
\hline 15 horas & $3,6 \%$ \\
\hline
\end{tabular}

Fonte: arquivo pessoal

Figura 2 - Boxplot da distribuição da carga horária diária de trabalho dos caminhoneiros na BR 101 - Casimiro de Abreu, 2018. 
Boxplot de Carga horária/ dia

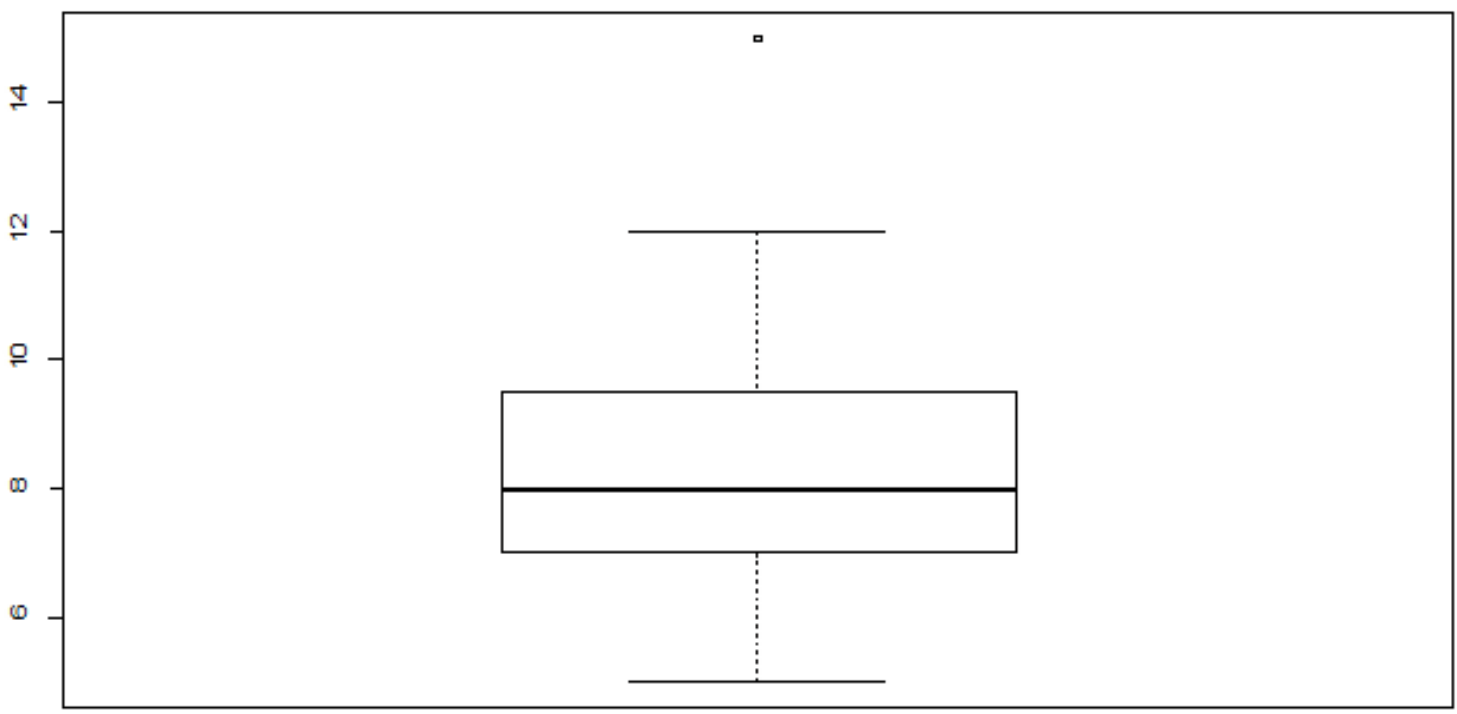

Fonte: arquivo pessoal

No que concerne aos acidentes de trânsito que ocorrem durante o percurso de trabalho, a maioria dos caminhoneiros que participaram do estudo relataram não ter sofrido acidentes em rodovias. Em relação ao transporte de produtos perigosos, quase $100 \%$ dos caminhoneiros não realizavam esse tipo de transporte (Tabela 4).

Tabela 3- Distribuição das variáveis relacionadas a acidente e tipo de material transportado pelos caminhoneiros, 2018.

\begin{tabular}{|l|l|}
\hline Acidentes em rodovias & Percentual \\
& $(\mathrm{N}=28)$ \\
\hline Sim & $32,1 \%$ \\
\hline Não & $67,9 \%$ \\
\hline Transporte de materiais perigosos & $\mathbf{( N = 2 8 )}$ \\
\hline Sim & $7,1 \%$ \\
\hline Não & $92,8 \%$ \\
\hline
\end{tabular}

Fonte: arquivo pessoal 
Em relação à saúde dos caminhoneiros participantes, foi investigada a questão da cobertura por plano de saúde, quadro de doenças crônicas e uso de substâncias lícitas. No contexto da cobertura por plano de saúde, a maior proporção dos caminhoneiros não possui plano de saúde, representando $71,4 \%$ participantes. Nas variáveis de doenças crônicas, $89,3 \%$ dos participantes relataram não ser portadores de diabetes e 85,7\% declararam não ser hipertensos. Quanto ao uso de substâncias lícitas, 89,3\% participantes apontaram não praticar o tabagismo (Tabela 4).

Tabela 4 - Distribuição das variáveis relacionadas a saúde dos caminhoneiros, 2018.

\begin{tabular}{|l|l|}
\hline Possui plano de saúde & Percentual \\
\hline Sim & $(\mathrm{N}=28)$ \\
\hline Não & $28,6 \%$ \\
\hline Diabetes & $78,4 \%$ \\
\hline Sim & $\mathbf{( N = 2 8 )}$ \\
\hline Não & $7,1 \%$ \\
\hline Não informou & $89,3 \%$ \\
\hline Hipertensão & $3,6 \%$ \\
\hline Sim & $\mathbf{( N = 2 8 )}$ \\
\hline Não & $10,7 \%$ \\
\hline Não informou & $86,5 \%$ \\
\hline Tabagismo & $3,6 \%$ \\
\hline Sim & $\mathbf{( N = 2 8 )}$ \\
\hline Não & $10,7 \%$ \\
\hline
\end{tabular}

Fonte: arquivo pessoal

\section{DISCUSSÃO}


O perfil dos caminhoneiros pesquisados não parece diferir de outros estudos nacionais, sendo na grande maioria homens caminhoneiros com renda mensal um pouco acima de 1 salário-mínimo ( $R$ \$998,00 até a publicação do presente estudo) e com carga horária de 8 horas de trabalho. Cabe ressaltar que na maioria dos casos, os motoristas de transportes formam a sua renda mensal por uma combinação de salário e comissão, o que caracteriza o motivo de muito trabalhadores optarem por conduzir caminhões. Contudo, tal modelo econômico afeta diretamente as suas condições de trabalho, motivo pelo qual muitos caminhoneiros relatam em estudos encontrados na literatura o desgaste sofrido por longas horas de trabalho (CNT, 2016; TRILICO, 2015; DELFINO, 2015).

Entretanto, os caminhoneiros que trabalham com uma carga horária diária acima do estabelecido em lei, no presente estudo, representou 17,9\% dos participantes, o que foi uma observação interessante, pois sugere a adoção da lógica da "lucratividade" pelos trabalhadores. Em outras palavras, quanto maior for a sua rapidez em chegar aos pontos de entrega e despachar as mercadorias designada ao proprietário, maior será o seu faturamento no trabalho, visto que realizarão um maior número de entregas. Entretanto, essa atitude pode exigir um aumento na carga horária semanal de trabalho através do menor tempo de descanso e da menor ocorrência de interrupção em longas distâncias percorridas, gerando um maior risco de ter sonolência e propiciando o consumo de estimulantes, seja legais ou ilegais (THOMPSON; STEVENSON, 2014).

Nesse contexto, de acordo com a pesquisa realizada por Hino et al (2017), a maioria dos caminhoneiros participantes de seu estudo informaram buscar o sistema de saúde, no atendimento de urgência e emergência, em situações de dores intensas e aguda. Esse cenário fortalece o modelo de saúde biomédico, focado na cura, o que adicionado ao fato de que possivelmente estarem trabalhando na lógica da lucratividade pode gerar vários prejuízos a saúde dos caminhoneiros.

Ainda citando os achados de Hino et al (2017), quanto a assistência à saúde, os seus achados vêm de encontro com os dados observados no presente artigo, pois uma 
parcela dos caminhoneiros avaliados relatara não possuir plano de saúde o que dificulta ainda mais o acesso aos métodos de prevenção. Já uma análise comparativa dos dados do atual estudo com os resultados apresentados por Ramos et al (2018), observando a saúde por outro enfoque, permite analisar que, em ambas as pesquisas, um percentual reduzido de caminhoneiros diagnosticados com hipertensão arterial sistêmica e diabetes mellitus foi observado. Inclusive Ramos et al (2018) detectaram nos caminhoneiros participantes do seu estudo um percentual de diagnóstico de 17,84\% para hipertensão arterial sistêmica e 9,73\% para diabetes mellitus, obtendo valores superiores de hipertensos e um valor aproximado de diabéticos quando comparados aos resultados encontrados no presente estudo.

A pesquisa realizada por Angeles et al (2014) referente a carência da atenção primária a saúde dos caminhoneiros revela que, embora um pequeno percentual dos caminhoneiros participantes do estudo tenha apontado certo histórico de doenças, tais como diabetes mellitus, doenças cardíacas e pulmonares, foi detectado uma elevada prevalência de fatores de risco para doenças crônicas e cardiovasculares. Entre os fatores de risco que foram encontrados a ingestão de sal diária acima da média recomendada e uma dieta pobre em nutrientes, receberam maiores destaques. Por conseguinte, ainda que os caminhoneiros participantes do atual estudo não apresentem uma prevalência significativamente alta de histórico de doenças crônicas, esse fato não deve descartar um elevado risco para quadros futuros. Portanto, é essencial que os caminhoneiros recebam um acompanhamento de saúde, principalmente do Sistema Único de Saúde, haja vista que em nosso estudo 71,4\% dos caminhoneiros não tinham plano de saúde.

Outro ponto a ser destacado é que o fato de os caminhoneiros estudados não portarem doenças crônicas, como a diabetes mellitus e a hipertensão arterial sistêmica, pode estar relacionado à falta de acompanhamento médico, o que dificulta um possível diagnóstico. Diversos caminhoneiros não buscam o sistema de saúde para consultas e essa situação está associada à rotina exaustiva de trabalho junto com os padrões sociais relacionados à masculinidade. Essa atitude é corroborada por uma pesquisa realizada em um Centro de Saúde no interior de São Paulo, em que os 
atendimentos aos homens estavam ligados ao tratamento de doenças crônicas, como a diabetes mellitus, hipertensão arterial sistêmica e doenças do trato respiratório. Contudo, os métodos de prevenção de doenças, tanto primárias quanto secundárias, foram adotados por menos da metade dos homens atendidos, revelando que a abordagem dos atendimentos à saúde do público masculino volta-se mais para a cura do adoecimento do que o uso de métodos de prevenção de doenças (BERTOLONI; SIMNETTI, 2014).

Quanto aos acidentes em rodovias e sua relação com a carga horária de trabalho, observou-se que mais da metade dos caminhoneiros que compõe o estudo relataram não ter sofrido nenhum tipo de acidente de trânsito no expediente de trabalho. Esse resultado pode estar relacionado ao fato de que quase metade dos caminhoneiros participantes trabalha 8 horas diária, não ultrapassando o limite exigido em lei. Segundo Fragosso et al (2019), entre os fatores que predispõe a ocorrência de acidentes de trabalho com motoristas de caminhão que percorrem longa distância, se encontram em destaque a elevada jornada de trabalho e a falta descanso vivenciados por esses profissionais.

\section{CONSIDERAÇÕES FINAIS}

A profissão de caminhoneiro é uma carreira que aumenta a vulnerabilidade dos trabalhadores a doenças e outros agravos, visto que as características específicas deste exercício laboral e seu ambiente de trabalho propiciam ao trabalhador a adotar maus hábitos alimentares e de descanso, além de favorecer o sedentarismo, o estresse e a redução da procura por um serviço de atenção primária a saúde. Em parte, as evidências apresentadas nesse estudo corroboram com as informações encontradas na literatura na medida em que foi observada a carga horária diária de trabalho e a ocorrência de acidentes de trânsito durante o ofício, duas situações extremamente relevantes para traçar o perfil da vulnerabilidade laboral dos caminhoneiros. Na teoria, o que se tem é que quanto menor a carga horária diária de trabalho, menor será o risco para a ocorrência de acidentes e vice-versa. 
Outro ponto relevante levantado no presente estudo foi a baixa deteç̧ão de diagnósticos de doenças crônicas. Entretanto, tal achado não traduz um estilo de vida saudável dos caminhoneiros entrevistados, o que supostamente é encoberto por uma pequena procura pelos serviços de saúde para o diagnóstico e um prognóstico de agravos a saúde gerados por um estilo de vida não saudável promovido por esta profissão. Portanto, sugere-se que sejam realizados mais estudos envolvendo esses trabalhadores para a apresentação de novas ações de promoção à saúde e bem-estar dos caminhoneiros, com vistas a reduzir as vulnerabilidades sociais desencadeadas pela profissão com amparo em evidência científica.

\section{REFERÊNCIAS}

ALESSI, Angélica; ALVES, Márcia. Keller. Hábitos de vida e condições de saúde dos caminhoneiros do Brasil: uma revisão da literatura. Revista Ciência e Saúde, v. 8. n. 3, p. 129-136, 2015. Disponível em: $<$ https://www.researchgate.net/publication/292076975_Habitos_de_vida_e_condicoe s_de_saude_dos_caminhoneiros_do_Brasil_uma_revisao_da_literatura>. Acesso em: 27/07/2020.

ANGELES, Ricardo et al. Primary health care needs for a priority population: a survey of professional truck drivers. Work, v. 49, p. 175-181, 2014. Disponível em: $<$ https://www.researchgate.net/publication/242332741_Primary_health_care_needs_f or_a_priority_population_A_survey_of_professional_truck_drivers>. Acesso em: 27/07/2020.

BETOLLINI, Daniele Natália Pacharone; SIMONETTI, Janete Pessuto. O gênero masculino e os cuidados de saúde: a experiência de homens de um centro de saúde. Revista Anna Nery, v. 18, n. 4, p. 722-727, 2014 . Disponível em: $<$ https://www.scielo.br/pdf/ean/v18n4/1414-8145-ean-18-04-0722.pdf>. Acesso em: 27/07/2020.

CNT. Confederação Nacional de Transporte. Pesquisa CNT de perfil dos caminhoneiros 2016. Brasília: CNT, 2016. 98 p. Disponível em: 
<http://cms.cnt.org.br/Imagens\%20CNT/PDFs\%20CNT/Pesquisa\%20de\%20Perfil\%2 0dos\%20Caminhoneiros/Pesquisa_CNT_de_Perfil_dos_Caminhoneiros_2016_Com pleto.pdf>. Acesso em: 27/07/2020.

CHEN, Guang. X. et al. Vital signs: seat belt use among long-haul truck drivers - united states, 2010. Morbidity and Mortality Weekly Report, v. 64, n. 8, p. 217-221, 2015. Disponível em: <https://www.ncbi.nlm.nih.gov/pmc/articles/PMC4584718/pdf/217221.pdf>. Acesso em: 27/07/2020.

COSER, Janaina et al. Triagem dos perfis lipídico de glicídico em caminhoneiros que trafegam no centro unificado de fronteira, entre Brasil e Argentina. Revista Brasileira de Analises Clinicas, v. 41, n. 3, p. 223-228, 2009.

DELFINO Livia Guimarães; MORAES, Thiago Drumond. Percepções sobre adoecimento para caminhoneiros afastados pelo sistema de previdência social. Revista Estudos Interdisciplinares em Psicologia, Londrina, v. 6, n. 2, p. 113-143, 2015. <http://www.uel.br/revistas/uel/index.php/eip/article/view/23932/17866>. Acesso em: 27/07/2020.

FRAGOSO JUNIOR, Ademar; GARCIA, Eduardo Garcia. Transporte rodoviário de carga: acidentes de trabalho fatais e fiscalização trabalhistas. Revista Brasileira de Saúde Ocupacional, v. 44, n. 3, p. 1-12, 2019. Disponível em: $<$ https://www.scielo.br/pdf/rbso/v44/2317-6369-rbso-44-e3.pdf>. Acesso em: 27/07/2020.

HINO, Paula et al. Análise dos cuidados à saúde dos caminhoneiros. Revista de Enfermagem UFPE online, Recife, v. 11, n. 11, p. 4741-4748, novembro, 2017. Disponível em<https://periodicos.ufpe.br/revistas/revistaenfermagem/article/view/231217/25231 >. Acesso em: 27/07/2020. 
MASSON, Valéria Aparecida; MONTEIRO, Maria Inês. Estilo de vida, aspectos de saúde e trabalho de motoristas de caminhão. Revista Brasileira de Enfermagem, Brasília, v. 63, n. 4, p. 533-540, 2010. Disponível em: $<$ https://www.scielo.br/pdf/reben/v63n4/06.pdf>. Acesso em: 27/07/2020.

RAMOS, Bruna Helena et al. Condições de vida, trabalho e saúde de motoristas de transporte de cargas. Revista de Enfermagem UFPE online, Recife, v. 12, n. 1, p. 150-159, 2018.

Disponível em: $<$ https://periodicos.ufpe.br/revistas/revistaenfermagem/article/view/22922/25967>. Acesso em: 27/07/2020.

OLIVEIRA, Tânia Modesto Veludo de. Amostragem não probabilística: adequação de situações para uso e limitações de amostras por conveniência, julgamento e quotas. Revista de Administração Online, v. 2, n. 3, p. 1-13, 2001. Disponível em: $<$ <ttps://pesquisa-eaesp.fgv.br/sites/gvpesquisa.fgv.br/files/arquivos/veludo__amostragem_nao_probabilistica_adequacao_de_situacoes_para_uso_e_limitacoes _de_amostras_por_conveniencia.pdf >. Acesso em: 27/07/2020.

THOMPSON, Jason; STEVENSON, Mark. Associations between heavy-vehicle driver compensation Methods, fatigue-related driving behavior, and sleepiness. Traffic Injury Prevention, v. 15, p. 10-14, 2014 . Disponível em: $<$ https://www.researchgate.net/publication/266797765_Associations_Between_Heav y-Vehicle_Driver_Compensation_Methods_FatigueRelated_Driving_Behavior_and_Sleepiness>. Acesso em: 27/07/2020.

TRILICO, Matheus Luis Castelan. et al. Discursos masculinos sobre a prevenção e promoção da saúde do homem. Revista Trabalho e Educação em Saúde, Rio de Janeiro, v. 13, n. 2, p. 381-395, 2015. Disponível em: $<$ https://www.scielo.br/pdf/tes/v13n2/1981-7746-tes-sip00015.pdf>. Acesso em: 27/07/2020. 
Enviado: Agosto, 2020.

Aprovado: Novembro, 2020. 